\title{
A quantitative test of Jones NTC beaming theory using CLUSTER constellation
}

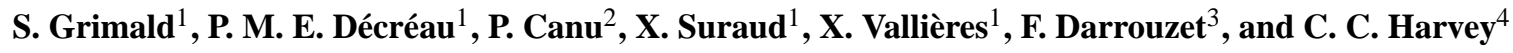 \\ ${ }^{1}$ Laboratoire de Physique et Chimie de l'Environnement (LPCE), Orléans, France \\ ${ }^{2}$ Centre d'Etude des Environnements Terrestre et Planétaires (CETP), Vélizy, France \\ ${ }^{3}$ Belgian Institute for Space Aeronomy (IASB-BIRA), Brussels, Belgium \\ ${ }^{4}$ Centre d'Etude Spatiale des Rayonnements (CESR), Toulouse, France
}

Received: 29 March 2006 - Revised: 8 January 2007 - Accepted: 13 February 2007 - Published: 29 March 2007

\begin{abstract}
Non-thermal continuum (NTC) radiation is, with auroral kilometric radiation (AKR), one of the two electromagnetic emissions generated within the Earth's magnetosphere and radiated into space. The location of the source of NTC has been sought for several decades, with only limited success. The constellation formed by the four CLUSTER spacecraft provides the possibility of triangulation in the vicinity of the source, thus allowing progress in source localisation, while simultaneously revealing the beaming properties of NTC radio sources.
\end{abstract}

We present a case event showing two beams localised on opposite sides of the magnetic equator. At any selected frequency, triangulation points to a single region source of small size. Its position is compatible with the range of possible loci of sources predicted by the radio window theory of Jones (1982) in a frame of constraints relaxed from the simple sketch proposed in early works. The analysis of similar observations from the Dynamics Explorer 1 by Jones et al. (1987) enabled the authors to claim validation of the radio window theory. CLUSTER observations, however, reveal a large beaming cone angle projected onto the ecliptic plane, a feature unobservable by Dynamics Explorer which had a different spin axis orientation. According to the radio window theory, such a large observed cone angle can only be formed by a series of point sources, each beaming in a narrow cone angle. This study demonstrates the difficulty of validating NTC linear generation mechanisms using global beaming properties alone.

Keywords. Magnetospheric physics (Plasmasphere) - Radio science (Radio wave propagation)

Correspondence to: S. Grimald

(grimald@cnrs-orleans.fr)

\section{Introduction}

Non-thermal continuum (NTC) radiation is an incoherent broadband electromagnetic radiation of low intensity and long duration which is observed in the frequency range from a few $100 \mathrm{~Hz}$ to several $100 \mathrm{kHz}$. It has been observed in the Earth's environment (Gurnett, 1975; Etcheto et al., 1982; Morgan and Gurnett, 1991; Kasaba et al., 1998; Décréau et al., 2004), as well as in the environment of other magnetized planets (Kurth, 1992). Terrestrial NTC is widely believed to be generated at the plasmapause, a region of strong density gradient, by conversion of electrostatic to electromagnetic waves. The electrostatic source has been suggested to be the intense UHR (Upper Hybrid Resonance) waves found at the geomagnetic equator and close to the plasmapause (Gough et al., 1979). The spacing between narrow spectral features has been related to the gyrofrequency at the source (Kurth et al., 1981). The implied underlying scenario is detailed by Gough (1982). Near the plasmapause, plasma frequency decreases rapidly with increasing radial distance, crossing several successive electron gyro-harmonic frequencies in only a short distance. It is assumed that strong electrostatic waves are produced, offset in frequency from each harmonic by a similar amount. Conversion of these waves to electromagnetic waves yields a series of narrow-band continuum radiation features, separated in frequency by approximately the electron gyrofrequency, which is quasi-uniform within the plasmapause layer.

Various mechanisms have been proposed to explain the wave conversion (Barbosa, 1982). The most developed theory is the linear one which supposes a conversion through propagation of the electrostatic UHR wave into an electromagnetic Z-mode wave (Jones, 1976). When aligned nearly parallel or anti-parallel to the ambient magnetic field, these Z-mode waves would produce NTC by conversion to the ordinary mode; this is the so-called "radio window" for waves at the local plasma frequency $F_{p e}$ (Budden, 1980; Jones,

Published by Copernicus GmbH on behalf of the European Geosciences Union. 


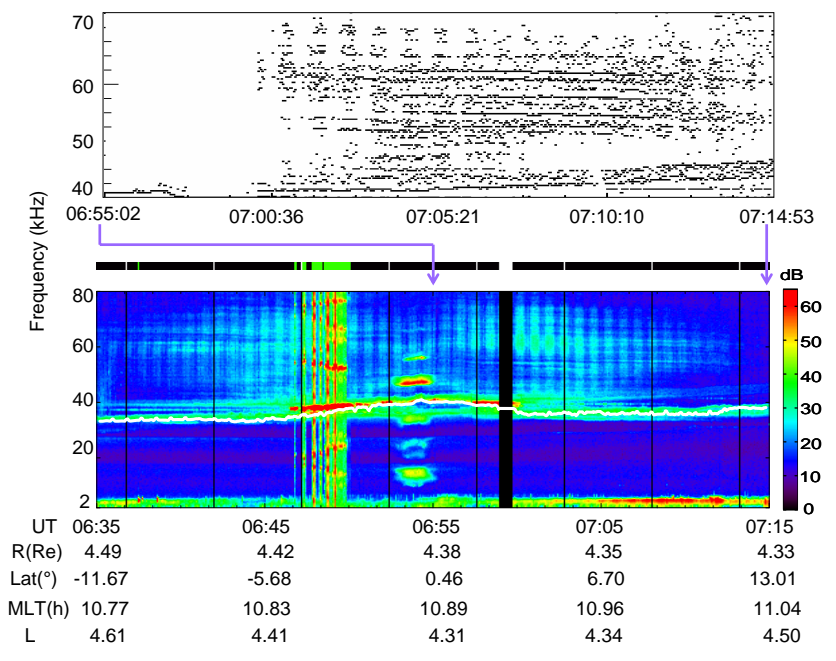

Fig. 1. Spectrograms of natural emissions observed on 26 September 2003 by the WHISPER instrument on board C3 satellite. Top: Frequency/time positions of intensity maxima in the $40-80 \mathrm{kHz}$ band. The lower maxima were suppressed (see text). Bottom: Frequency/time spectrogram in the $2-80 \mathrm{kHz}$ frequency band. The white line indicates the position of local plasma frequency, and the bar above the spectrogram shows the status of each spectrum (white: data gap; black: clean spectrum; green: saturated spectrum).

1980). Thereafter the strong density gradient at the plasmapause causes these two ordinary mode waves, initially at the local plasma frequency and propagating nearly parallel and anti-parallel to the magnetic field, to be refracted outwards to form two beams located symmetrically with respect to the magnetic equator in the plane through the source containing the density gradient and the magnetic field. As they propagate to regions of lower density the inclination angle $\alpha$ of these beams with respect to the magnetic equatorial approaches:

$\alpha=\operatorname{atan} \sqrt{F_{c e} / F_{p e}}$,

where $F_{c e}$ (electron cyclotron frequency) and $F_{p e}$ (electron plasma frequency) are defined at the source.

Such a quantitative property is extremely useful because it allows us to link a given NTC observation (position of the observatory and frequency of the wave) to the locus of the positions of candidate sources, thus remotely sensing the topology of active regions. Promoting the linear radio window theory, Jones (1983) developed the remote sensing approach, and even treated the problem of sources in movement. As long as the derived source positions and movement are realistic and in particular when the results agree with source localisation derived from directivity measurements, the observations have supported the radio window conversion theory (see Jones et al., 1987, for a claim of validation of the theory). However, other experimental observations suggest that the simple "single source - two beams" picture is too simplistic. For example, Morgan and Gurnett (1991), in a statistical study using Dynamics Explorer 1 (DE1) data, have shown that the existence of two symmetrical beams is very rare, suggesting that asymmetries in the density profile or in the magnetic field may be responsible. Furthermore, another important feature of NTC observations, the presence of a series of very narrow band structures more closely spaced than $F_{c e}$, has led to the proposal of another generation mechanism: strong upper hybrid turbulence (Christiansen et al., 1984).

In this paper, we analyse a "double beam" case event observed with the CLUSTER constellation, similar to the DE1 case event analysed by Jones et al. (1987). We discuss not only beaming properties, but also details of the spectral structure. Compared to a single spacecraft, the four CLUSTER satellites offer major advantages: spectral characteristics viewed from the different platforms can be compared, and spatial/temporal variations disentangled. We present the observations, and then (Sect. 2) use the approach which Jones et al. (1987) applied to DE1 data to examine where NTC sources would be located according to the radio window theory (Sect. 3). Finally, we discuss the quality and limitations of the test, as well as other findings derived from this study (Sect. 4).

\section{Observations}

\subsection{CLUSTER constellation and data}

The CLUSTER mission consists of four identical satellites (which we denote here by C1, C2, C3 and C4) in a tetrahedral configuration. They travel in a near-polar orbit with perigee located at $\sim 4.5 R_{E}$ near the ecliptic. On 26 September 2003, the inter-spacecraft separation distances lay between $\sim 300 \mathrm{~km}(\mathrm{C} 2-\mathrm{C} 3)$ and $1200 \mathrm{~km}(\mathrm{C} 1-\mathrm{C} 4)$; near perigee, where the CLUSTER configuration is elongated along the direction of the orbital velocity. The four CLUSTER satellites crossed the magnetic equator between 06:40 and 07:00 UT at about 11:00 MLT.

Observations presented in this paper are derived mainly from the WHISPER instruments, four sounders which measure the naturally occurring electric field in the frequency range $(2-80 \mathrm{kHz})$ when in passive mode of operation, and the characteristic frequencies of the plasma in the range (2$80 \mathrm{kHz}$ ) when in active mode of operation (Décréau et al., 2001). Each satellite measures the electric field with a double sphere antenna spinning in a plane nearly parallel to the ecliptic. The bottom part of Fig. 1 presents a frequencytime spectrogram of electric field measured by WHISPER in its passive mode on board $\mathrm{C} 3$. The time interval shown, 06:35-07:15 UT, includes the phenomenon of interest, i.e. NTC emissions, present on both sides of the equator, at frequencies above the plasma frequency $F_{p e}$, indicated by a solid white line. At about 06:53 UT C 3 crossed the magnetic 
equator, where localised intense electrostatic emissions are seen at frequencies $(\mathrm{n}+1 / 2) F_{c e}$ lying below $F_{p e}$. Such emissions, first observed on board OGO 5 (Kennel et al., 1970), co-exist in this event with equatorial emissions above $F_{p e}$ at the Bernstein frequencies $F_{q s}$ (Bernstein, 1958); this identification is confirmed by the sounder in active mode. The latter emissions could be a primary (electrostatic) source of NTC, invoked in the nonlinear decay generation mechanism proposed by Rönnmark (1985). Additional intense emissions are observed on each side of the magnetic equator, just above the plasma frequency (near upper hybrid frequency); they appear to be intensifications of a signal at the upper hybrid frequency which is present nearly continuously between $06: 43$ and 07:00 UT. The southern intensification occurred around 06:49 UT and the northern one around 06:58 UT. During the first enhancement, the receiver saturates, creating spurious replicas (roughly $10 \mathrm{kHz}$ apart) of the real emission which was at about $38 \mathrm{kHz}$. These intense emissions observed near plasma frequency in the plasmapause layer on each side of the magnetic equator are the other candidates for primary sources of NTC.

NTC emissions are seen in the frequency range 45$75 \mathrm{kHz}$. They display a periodic time variation, due to the spin modulation of the signal. Actually, the apparent period is a stroboscopic effect due to the combination of a half-spin period $(\sim 2 \mathrm{~s})$ with the sequencing period of instrument's operations (52 s). In addition, the emissions are structured in narrow frequency bands, stable in frequency and very slowly varying in time. To compensate for the stroboscopic effect, each frequency spectrum obtained during a single acquisition interval has been normalized to the dynamical spectrum covered by the spectrum (after this treatment, all spectra present intensities varying between the same two levels, minimum and maximum). Then, in each spectrum, all intensity maxima (frequency peaks) have been identified, and only those placed above a chosen intensity level have been selected. The result is displayed in the top panel of Fig. 1. This treatment highlights a series of quasi-monochromatic emissions (horizontal lines) of a few minutes duration, appearing, for example, at about 54.7, 57.8, 60.6 and $62.0 \mathrm{kHz}$ near 07:08 UT on C3. This detailed frequency pattern is identified at exactly $( \pm 180 \mathrm{~Hz})$ the same frequencies and the same time $( \pm 2 \mathrm{~s})$, on all four spacecraft. This indicates that, whether or not all frequencies are produced by the same source, the beams are wide enough to illuminate all four spacecraft. However, the different satellites are illuminated at different intensities, indicating a finite beam size, as discussed below.

\subsection{Derived quantities}

Assuming that this NTC radiation is electromagnetic and propagating in the $\mathrm{O}$ mode (quasi-circular polarisation), the spin modulation can be used to determine the projection of the direction of propagation onto the antenna spin plane (Calvert, 1985; Gurnett, 1975; Gough, 1982; Kasaba et al.,

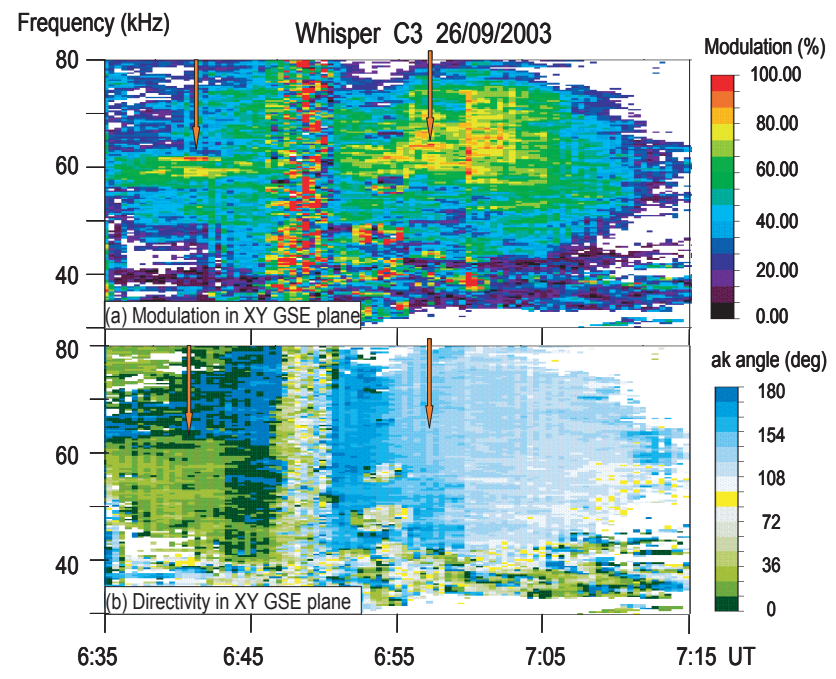

Fig. 2. Parameters derived from spin modulation of electric field intensity, plotted as frequency/time spectrograms: (a) modulation index $\mathrm{m}$; (b) directivity angle ak. The orange arrows point to the high modulation regions.

1998; Décréau et al., 2004). Figure 2 presents the frequency/time evolution of the two parameters which are derived from this process. The upper panel (a) displays the modulation index $\mathrm{m}$ (ratio of modulated to total power) and the lower panel (b) the directivity angle ak (angle between X GSE axis and ray path direction, projected onto the XY GSE plane, where GSE refers to the geocentric solar ecliptic system). Occurrences of the high modulation index $(m>73 \%$, indicated by orange arrows) are visible on both sides of the equator, between 57 and $62 \mathrm{kHz}$ in the Southern Hemisphere and between 57 and $77 \mathrm{kHz}$ in the Northern Hemisphere. They present a stratified structure, like the frequency peaks in Fig. 1. The frequencies of the lines of the high modulation index correspond to those peaks in the spectral power. Note that the frequency/time occurrence of a high modulation index does not correspond to any well-delimited region with specific ak angles in Fig. 2b. In other words, the source of the less modulated emissions appears to be located in the same general direction as the source of the highly modulated emission.

When more than one satellite is available, the projections onto the spin planes of the various ray paths can be used to estimate source positions by triangulation (Gough, 1982; Décréau et al., 2004). If the source is stable with time, a single spacecraft can provide the information using ray paths at different times (Morgan and Gurnett, 1991). Only a 2-D picture of directivity can be achieved from such measurements in the XY GSE plane, as from CLUSTER. Figure 3 displays the orbits of $\mathrm{C} 2$ and $\mathrm{C} 4$ projected into this plane. The source directions for the emission at $70 \mathrm{kHz}$ are drawn (in blue) from C4 in the Southern Hemisphere and (in red) from C2 in the Northern Hemisphere. The other spacecraft provide 


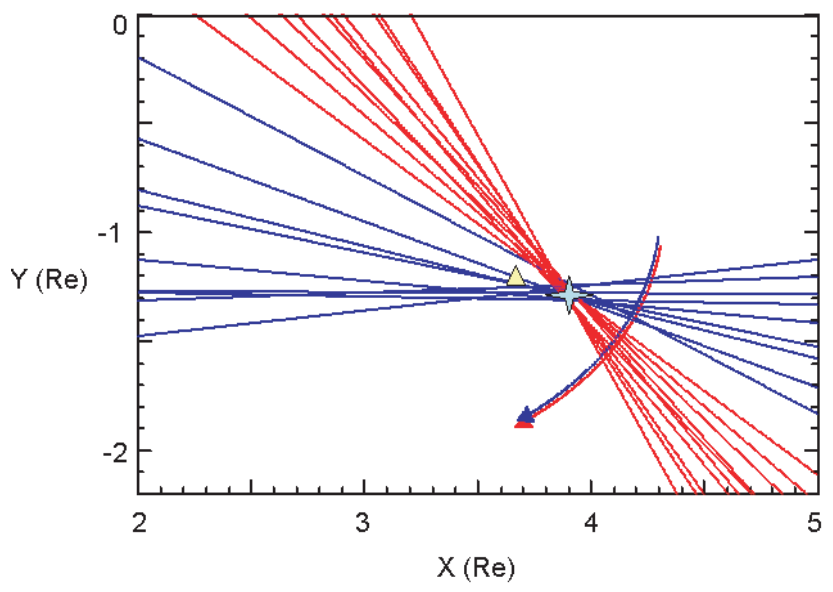

Fig. 3. Triangulation in XY GSE plane. Directivity lines drawn from $\mathrm{C} 4$ in the Southern Hemisphere (blue lines) and from C2 in the Northern Hemisphere (red lines) for the $70-\mathrm{kHz}$ emission indicate a source (grey star) further from the Earth than the source position estimated according to the radio window beaming theory (yellow triangle). The arrows give the trajectories of $\mathrm{C} 2$ and $\mathrm{C} 4$.

similar information in both hemispheres. A source direction is plotted only when the modulation index is large enough $(\sim 40 \%)$ to determine the ak angle with a precision of $\pm 2^{\circ}$. It is remarkable that all the lines cross in the same region, thus pointing to a source region stable over the total time interval (06:35-07:15 UT). This source region, indicated by the grey star, is small, about $0.25 R_{E}$ in diameter in the equatorial plane, located at about $4.15 R_{E}$ from the $\mathrm{Z}_{\mathrm{GSE}}$ axis. In fact, the triangulation technique defines only a column perpendicular to the common spin plane. The $\mathrm{Z}_{\mathrm{GSE}}$ coordinate of the source within this column is uncertain. Amongst other factors, the modulation index is determined by the inclination angle of the ray with respect to spin plane, and this provides a crude indication about the $\mathrm{Z}$ position of the source. But the uncertainty of such an estimate is too large, however, to allow for localization in 3-D. In any case, the 2-D result obtained can be compared to source positions estimated from the theory. Finally, we note that projected directivity lines cover a large range of azimuth angle, of $60 \mathrm{deg}$ or more when combining both hemispheres. This provides an estimation of the cone angle size, projected onto the XY GSE plane, of the double beam emitted from the source region. We will return to this.

\section{Test of Jones theory}

\subsection{Basic beaming configuration}

The first approach we have followed is to test the simple configuration proposed by Jones (1981), summarized in Sect. 1 and sketched in Fig. 4. The hypotheses used are:

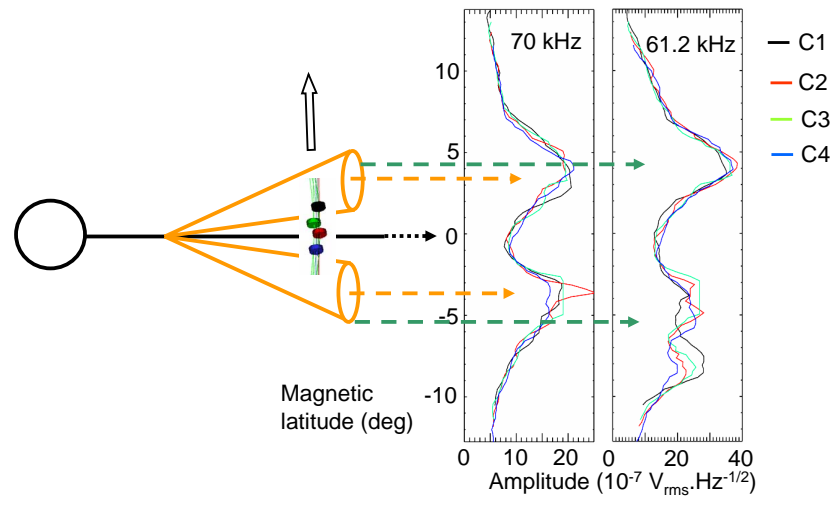

Fig. 4. Left: NTC beams (orange) and constellation configuration at perigee; the arrow denotes the direction of motion. Right: latitudinal profiles (in SM coordinates) of the electric field amplitude measured from the four CLUSTER platforms at $70 \mathrm{kHz}$ and $61.2 \mathrm{kHz}$. The colour coding is shown on the right.

A radio window is located in the equatorial plane of a dipolar magnetic field, in a smooth radial density gradient; and it radiates two narrow beams inclined symmetrically with respect to the equatorial plane, at an angle satisfying Eq. (1).

A second important constraint, which we will call here condition (2), is that the two principal ray paths are located in the plane containing the local magnetic field $\mathbf{B}$ and the density gradient vector $\nabla \mathrm{N}$ at the source. Actually, it is in the frame of this second constraint that Eq. (1) is derived.

A third hypothesis is that $\nabla \mathrm{N}$ is along the local radial axis, so that the ray paths are in the magnetic meridian plane, with $\mathrm{B}$ being aligned along the $\mathrm{Z}$ axis.

Fourthly, the spacecraft making the observations are assumed to travel in this meridian.

The panels on the right of Fig. 4 display the electric field amplitude at two frequencies, as determined on all four satellites, as a function of latitude in the solar magnetic (SM) system, which is close to the simple dipole geometry of the theory. At $70 \mathrm{kHz}$ each satellite observed two maxima, one in each hemisphere, and a minimum near the magnetic equator. The amplitude peaks are almost equidistant in magnetic latitude $\left(3.94^{\circ} \pm 0.5^{\circ}\right)$ from the magnetic equator and, to a first approximation, the satellites really do observe two beams symmetrically placed with respect to the equator. Note that the amplitude seen by $\mathrm{C} 3$ has been capped above $2 \times 10^{-8} \mathrm{~V}_{\text {rms }}$. $\mathrm{Hz}^{-1 / 2}$, to take account of amplifier saturation when $\mathrm{C} 3$ encounters intense electrostatic emission (as in Fig. 1).

As indicated in Fig. 4, C1 is the first satellite of the constellation to cross the beams, while $\mathrm{C} 4$ is the last. The four amplitude profiles are very similar, which indicates that the structure did not evolve significantly between their observation by $\mathrm{C} 1$ and by $\mathrm{C} 4$, that is, a time delay of about $3.5 \mathrm{~min}$. The latitudinal width of the beam at half intensity, $\sim 5^{\circ}$, is larger than the $2.25^{\circ}$ latitudinal separation between $\mathrm{C} 1$ and 
$\mathrm{C} 4$; this is consistent with the observation, already noted in Sect. 2.1, that a given spectral detail can be observed simultaneously by the complete constellation.

Latitudinal electric field amplitude profiles at other frequencies also show evidence of a double beam configuration. The two beams tend to move away from the magnetic equator with decreasing frequency, as illustrated in the right panel of Fig. 4. This is predicted by Eq. (1): the beaming angle depends upon the ratio $F_{c e} / F_{p e}$, at the radio window, which is where $F_{p e}$ is equal to the frequency of the observed NTC. Furthermore, $F_{c e}$ variations are small compared to $F_{p e}$ variations in the plasmapause region. Hence, emissions at higher frequencies should propagate at smaller inclination angles, and this is indeed the observed trend, except that it is no longer true at the lowest frequencies, where the double beam picture is only a crude approximation. For example, in Fig. 4, at $61.4 \mathrm{kHz}$, the southern beam splits into two parts which, indeed, are seen in different directions (the directivity angles in XY GSE plane differ by $\sim 20^{\circ}$ (not shown)). Such behaviour indicates the presence of two active sources at different MLT positions. We will now examine the radiation at $70 \mathrm{kHz}$, which presents a symmetric double beam which is easier to compare with theoretical expectations.

Following Jones (1981) and equations therein, we have calculated the radial position $R_{S}$ of the $70 \mathrm{kHz}$ radio window from the position of the satellite when the peak of intensity at this frequency was observed: geocentric distance $R_{C}=4.36 R_{E}$ and geomagnetic latitude $\lambda=3.4^{\circ}$. In practice, we have slightly modified Jones' equation: we still use a dipolar model of the magnetic field, but instead of using the gyrofrequency $f_{0}$ at the Earth's surface as the reference value, we use the gyrofrequency $f_{C C}$ measured at the satellites. The calculation finally leads to $R_{S}=3.8 R_{E}$, with $\alpha=24.5^{\circ}$ and $f_{C S}=14.5 \mathrm{kHz}$, where $f_{C S}$ is the gyrofrequency at the radio window. The field at this source location derived from a standard (Tsyganenko, 87) model of the magnetic field is $f_{C S}=14.45 \mathrm{kHz}$, in good agreement.

The source position, as derived above and projected onto the XY GSE plane, is indicated by the yellow triangle in Fig. 3. It is significantly closer to the Earth than the region defined by the triangulation of all rays received at $70 \mathrm{kHz}$. Furthermore, we note that the radial value $R_{S}=4.15 R_{E}$ which when projected onto XY GSE, would coincide with the source region derived from triangulation, would require a larger beaming angle, $\alpha=51^{\circ}$. Such a high value would lead, from Eq. (1), to a local (at the window) gyrofrequency of $107 \mathrm{kHz}$, which is higher than the frequency of observation and incompatible with the radio window model. Moreover, the difference between expected and observed radial values $\left(\sim 0.35 R_{E}\right)$ is too large to be attributed to the slight distance between the radio window and the intersection of the two emitted beams (see Fig. 4 of Jones, 1980). This distance, $\Delta \mathrm{S}$, linked to the beam curvature of the $\mathrm{Z}$ wave in the density gradient near the source, would place the real radio window source somewhat further from the Earth than our estimation.
The value $\Delta \mathrm{S}$ depends upon the actual density gradient in the plasmapause layer. By reference to a modelled plasmapause density profile (Carpenter and Anderson, 1992), and assuming, as shown in ray paths calculated by Jones (1976), that the beam undergoes its total deviation within a layer where the normalized square plasma frequency $\mathrm{X}$ varies between 0.9 and 1.4 (the density undergoing a variation of $50 \%$ ), we obtain $\Delta \mathrm{S}=0.025 R_{E}$. This is effectively negligible when compared to $0.35 R_{E}$.

This first test demonstrates that the linear radio window theory cannot be validated using such a simple geometrical configuration. It is necessary to relax two assumptions: (i) that sources and satellites are located in the same magnetic meridian plane; (ii) that sources are in the equatorial plane. We will now test the beaming angle Eq. (1) by successively relaxing each of these assumptions, under the guidance of Jones published work.

\subsection{Complex beaming configuration}

Jones (1982) generalized the remote sensing approach by expressing a visibility equation defining the loci in MLT and radial position of equatorial sources which would illuminate, at the centre of their beam, a spacecraft at a given position. This visibility equation incorporates the beaming condition of Eq. (1) and the condition (2), but conditions (3) and (4) are partially relaxed: the density gradient $\nabla \mathrm{N}$ at the source, no longer necessarily in the meridian plane, remains coplanar with the satellite-source line and the magnetic field at the source. The top two panels of Fig. 5 display such visibility contours, which are the loci of source positions in the equatorial plane which have the CLUSTER satellites at the centre of their $70 \mathrm{kHz}$ beam, respectively, in the Northern (Fig. 5a) and Southern (Fig. 5b) Hemispheres. The four visibility contours are almost identical, as expected for such small interspacecraft separations. The green cross represents the position of $\mathrm{C} 3$ projected onto the equatorial plane, and the dashed black line the magnetic meridian plane at that position. The source plane determined by direction finding at times of peak amplitude can be used to locate the source on the visibility contour; unfortunately, near peak amplitude the directivity determination is relatively poor $\left( \pm 5^{\circ}\right)$ and provides only mediocre directional information. Nevertheless, the results indicate that the sources are not in the meridian plane of the spacecraft (10.9 MLT), but at a later local time (11.2 MLT) for northern beam, and at an earlier one (10.8 MLT) for southern beam, strongly suggesting the existence of two separate sources. This may be compared with Fig. 5d, which shows latitude and longitude of C3 and C4 (which hide, respectively, C2 and C1) when these spacecraft were illuminated by NTC beams. The magnetic field vector measured at the equator is shown as an orange arrow. The split of the beams between two different local time sectors corroborates their split into two different hemispheres; there is no overlapping in MLT range, and none in latitudinal range. The 

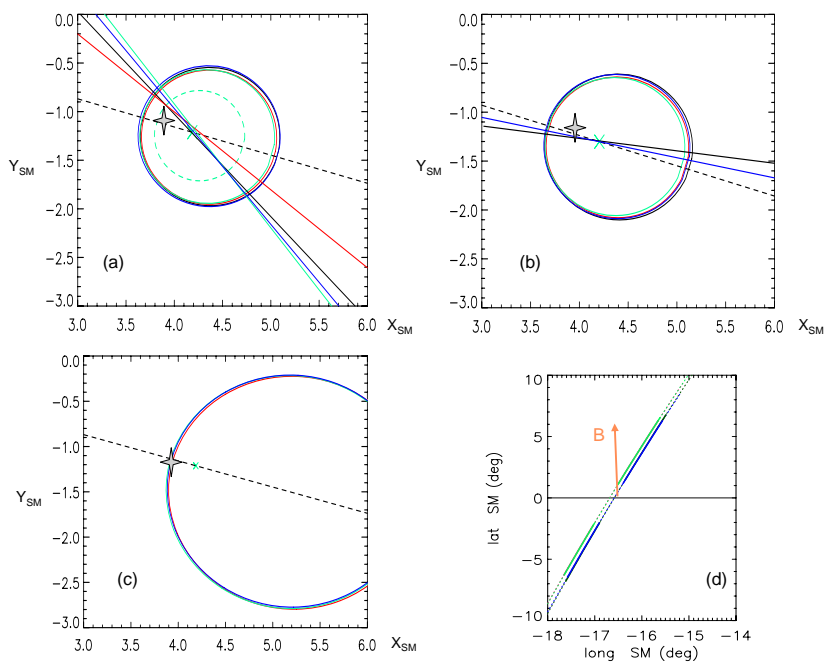

Fig. 5. Geometrical results for $70 \mathrm{kHz}$ radiation. Top: loci in the equatorial plane of sources which would have the Cluster $\mathrm{Cn}$ spacecraft, at the time it observes maximum amplitude at $70 \mathrm{kHz}$, at the centre of their beam, and the corresponding directivity paths (straight lines); colour codes for $\mathrm{Cn}$ as in Fig. 4. Panel (a) northern beam (dashed green line: the locus and directivity for $\mathrm{C} 3$ if it were $1^{\circ}$ latitude closer to the SM equator) and panel (b) for the southern beam. The green cross represents the position of $\mathrm{C} 3$, and the dashed black line the projected magnetic meridian plane at that position. The grey star symbol shows the source position obtained by triangulation and plotted in Fig. 3. Bottom: (c) loci in XY plane of an off equatorial source $\left(+0.65^{\circ}\right.$ latitude); (d) orbital positions of the four spacecraft and measured magnetic field orientation at equator (orange vector). Thick lines indicate the SM latitude and longitude of $\mathrm{C} 3$ and $\mathrm{C} 4$ when the signal at $70 \mathrm{kHz}$ was greater than half the peak amplitude.

two peaks in electric field amplitude are encountered at 10.85 and 10.92 MLT $\left(-17.3^{\circ}\right.$ and $-16.2^{\circ}$ longitude), respectively, in the Southern and Northern Hemispheres. Those positions are qualitatively in accordance with source MLT positions shown in Figs. 5a and b, but quantitatively at a significantly smaller MLT separation. Moreover, the source position deduced from triangulation (grey star) is still at a larger geocentric distance than is compatible with the visibility contours.

The first conclusion of this study is that the apparent source position is incompatible with the positions allowed by the linear radio window theory. However, the discrepancy may not be so significant when we take into account the measurement uncertainties. The uncertainty in the triangulated source longitude is about $0.12 R_{E}$. The uncertainty in the latitude of the observed peak intensity can be evaluated to $\pm 1^{\circ}$ (from Fig. 4) and leads to an uncertainty in the derived radial position of about $0.2 R_{E}$. This is illustrated in Fig. 5a, which displays the visibility curve for a spacecraft placed at a latitude $1^{\circ}$ below that of the estimated peak. A second conclusion is that the visibility of the sources outside the meridian plane must be considered, that is, longitudinal beaming properties. Such a discussion is necessary by the following critical observational fact: the comparison of the size of each illuminated orbit elements $\left(\sim 0.4 R_{E}\right)$ with the distance from source to satellite position $\left(\sim 0.6 R_{E}\right)$, indicates that the beaming angle is large $\left(\sim 40^{\circ}\right)$. Such a large value when the radio window theory predicts angular beam widths of about $2^{\circ}$ (Jones, 1982) indicates that irregular density gradients and perhaps spatially extended sources should be considered.

We examine further the observed directivity pattern (Fig. 3) in light of the second beaming condition. In a simple axi-symmetric plasmasphere model, the planes defined by $\mathbf{B}$ and $\nabla \mathrm{N}$ at equatorial plasmapause (the radio window) are meridians. In an axi-symmetric plasmasphere the only visible part of the active equatorial plasmapause layer is in the local meridian of the CLUSTER spacecraft. Thus, the measured directions of arrival, being part of successive meridians, would all point to the centre of the Earth. That the observed intersection (grey star, Fig. 3) is clearly not at the Earth's centre does not rule out the radio window theory. For example, we can abandon the axi-symmetric plasmasphere model.

CLUSTER travels eastward. The ray paths point initially (when below the equator) to a sector earlier than the local meridian, and later (above the equator) to a later sector. This can be realized either by a hollowed or by a bulged shape plasmapause surface. In case of a hollow, the individual sources would appear slightly closer to Earth than the focus point, and in case of a bulge they would appear slightly closer to spacecraft. Undulations of the plasmapause surface are necessarily associated with the density irregularities commonly observed in the plasmapause layer (Darrouzet et al., 2004), with typical dimensions of a few hundred $\mathrm{km}$. Moreover, those irregularities have been observed by the Cluster constellation to be field aligned (Décréau et al., 2005), ruling out undulations along the north-south direction. In our chosen event, a cross-field undulation could be present but undetectable by CLUSTER, because all spacecraft travel outside the main plasmasphere body. A hollowed surface would explain all or part of the discrepancy between radial distances of ray paths intersection and of expected radio window positions. This is the configuration retained for further discussion.

To conclude this discussion, we examine two possible interpretations of our observations:

1. A common point source is responsible for illuminating a large segment of the orbit. This requires that the beam cone is very large, at odds with beaming properties of the radio window theory,

2. A series of point sources are seen successively by the travelling spacecraft. These sources are embedded in an undulating plasmapause surface, normal to the local density gradient. Each source emits a narrow beam according to radio window theory, the centre of the beam 
being inclined with respect to the density gradient at an angle satisfying Eq. (1), in the plane containing the magnetic field vector and the surface normal vector. As central beams cover, a large angle range, together with surface normals, they illuminate a large orbit element. The high- and low-latitude limits of the illumination would be due in such a case to the actual limit - in latitude or longitude - of the overall emitting surface, combined to beaming effects.

According to the second scenario, the distance between the emitting surface and the focus point would be proportional to the radius of curvature of the surface. A concave surface could, in particular, explain some or all of the distance between the apparent source located by triangulation and the equatorial sources, closer to Earth, indicated by latitudinal beaming. A quasi-cylindrical hollow (aligned with the magnetic field) of radius $\sim 0.3 R_{E}$ would explain our estimation of the observed distance.

We have, however, no practical means to evaluate the real dimensions of plasmapause surface undulations in this case event and, assuming that its radius of curvature is small, we perform the last step of our quantitative test. We relax the constraint on the latitude of the source. We have already noted (Sect. 2) the likely crossing by $\mathrm{C} 3$ of non equatorial sources (f $\sim 38 \mathrm{kHz}$ and $\lambda \sim+/-2.5^{\circ}$ latitude). Similarly, in his model to fit the DE1 observations, Jones et al. (1987) relaxed the constraint of the source being in the equatorial plane. The DE1 spin plane was near the meridian, and hence the 2-D source position estimations via directivity were in this plane. The DE1 observations point to sources located off the equator. Assuming a similar situation for our event at $\mathrm{f}=70 \mathrm{kHz}$, by assigning a positive latitude at the source we have modified the equations to obtain visibility contours projected in the $\mathrm{XY}(\mathrm{SM})$ plane similar to the generalized remote sensing contours displayed in Jones (1983). By varying the source latitude, we obtain visibility contours (Fig. 5c) which perfectly match the source position (grey star) obtained from the triangulation. The corresponding magnetic latitude, $\lambda_{S}=0.65^{\circ}$, is realistic, corresponding to a source distance above the equator similar $\left(\mathrm{Z}_{S} \sim 0.05 R_{E}\right)$ to that observed by DE1. We emphasise that the visibility contours are very sensitive to the value of $\lambda_{S}$. To complete the picture, a complementary study should be conducted in the Southern Hemisphere or better yet, the analysis should be conducted separately in each hemisphere, starting from the source localisation by triangulation. However, the contours shown in Fig. $5 \mathrm{c}$ are sufficient to demonstrate that a source location satisfying Eq. (1) can be found.

The two points which emerge from this third step can be summarised thus:

1. We found a source position satisfying the first beaming property of radio window theory (Eq. 1) and matching the source position deduced from triangulation based on 2-D directivity properties measured by CLUSTER.
The assigned source position in $\mathrm{Z}$ is small, as compared to the diameter of the source region in XY GSE plane determined by triangulation. Since calculated visibility contours are very sensitive to the distance from the source to equatorial plane, relaxing the condition of an equatorial source opens so largely the domain region of source positions satisfying Eq. (1) that, in the absence of experimental 3-D source positioning, our test is not fully conclusive.

2. The second beaming property (beam coplanar with magnetic field vector and gradient density at the source) must also be satisfied. As discussed above, this property can only be satisfied under the assumption of multiple sources placed over a surface of a small radius curvature, possibly placed at a larger distance than the column highlighted by triangulation, and in addition, possibly placed at various positions in Z GSE.

The practical conclusion is that the source positions constrained by the two beaming conditions of the NTC radio window theory are compatible with our observations (at $70 \mathrm{kHz}$, in this particular case), provided that multiple sources are being seen on curved plasmapause surface and located slightly above the equator.

\section{Summary and conclusion}

We have analyzed an NTC emission event observed by the CLUSTER constellation when near perigee in the dayside MLT sector. This study has produced new results concerning the experimentally derived properties of NTC radiation in the vicinity of the source, and has led to an evaluation of the validity of the radio window theory of NTC generation.

Experimentally observed NTC properties concern successive intensity, spectral features, beaming and directivity:

1. Intensity

- The overall duration of the NTC emissions observed in the 35 to $75 \mathrm{kHz}$ frequency range was about $40 \mathrm{~min}$, during which time there were distinctly different periods situated along two orbital segments of length $\sim 0.4 R_{E}$, located on opposite sides of the geomagnetic equator,

- the emissions were stable for at least the time ( $\sim 3.5 \mathrm{~min}$ ) between crossings of a given position by the two most widely separated $\left(\sim 0.15 R_{E}\right.$ along the orbital direction) satellites of the constellation.

\section{Spectral features}

- Spectral analysis with 320-Hz frequency resolution indicates that the emissions consist of a series of narrow, closely spaced bands whose frequencies are varying very slowly. 


\section{Beaming}

- The two periods of signal intensification on opposite sides of the equator correspond to the satellites crossing two spatial structures, that is, two NTC beams bounded by either latitudinal or local time boundaries,

- at higher frequencies the observed NTC emission showed clear, smooth enhancements on both sides of the equator, but at lower frequencies more complicated variations (three or more signal intensifications) were observed south of the equator,

- when the constellation is near the centre of one of the beam structures, specific detailed spectral signatures are observed simultaneously by all four spacecraft.

\section{Directivity}

- Directivity angles measured in the spin plane of each spacecraft were, like the signal intensity, mainly controlled by the position of observation (relative to the stable "beam" structures), rather than by the time of observation,

- globally (over the entire frequency range) directivity angles varied gradually but significantly with spacecraft position, in such a way that ray paths point to a single apparent source region. We noted, however, that when crossing the equatorial plane, directivity angles changed by $\sim 20^{\circ}$, indicating a global change of behaviour from south to north,

- modulation indices confirmed the presence of narrow band frequency structures and that each of the two (Southern and Northern) large-scale structures exhibit different detailed features.

The implications of these observed properties as a test of the radio window beaming theory can be summarized as follows:

1. At frequencies high enough for the double peak configuration to be clear, latitudinal beaming increases with decreasing frequency, as expected. The equatorial source position derived quantitatively in the frame of the simple sketch of Jones' beaming theory (equatorial source and source-satellite line in the meridian plane) does not match the position derived from triangulation.

2. The assumption of a single longitudinal beaming angle from a single point source does not fit the observed directivity pattern. Compatibility with the radio window theory would require at least a scenario with the source region being an ensemble of small sources distributed over an undulating plasmapause surface, hence widely opening the longitudinal beaming angle.
3. Our refined estimation of source positions is compatible with triangulation results. We conclude that radio window theory for NTC generation is compatible with quantitative observed beaming properties.

This does not close the debate over which generation mechanism produces NTC radio emissions. The complex experimental context described above, as well as instrument limitations, do not allow us to constrain the source position within a sufficiently small volume of space for the test to be conclusive. The radio window theory is as yet neither validated nor invalidated. Further event studies, using different configurations of the CLUSTER constellation, may possibly provide more of a conclusive test. In addition, other points of the radio-window theory, such as its efficiency, need to be discussed, and other generation mechanisms also need to be tested against the Cluster data.

Acknowledgements. We would like to thank the teams at ESOC (Darmstadt), JSOC and Sheffield (UK) for their constant support of CLUSTER operations.

Topical Editor I. A. Daglis thanks M. P. Gough and O. Santolik for their help in evaluating this paper.

\section{References}

Barbosa, D. D.: Low-level VLF and LF radio emissions observed at earth and Jupiter, Rev. Geophys. Space Phys., 20, 316-334, 1982.

Bersntein, I. B.: Waves in a plasma in a magnetic field, Phys. Rev., 109, 10-21, 1958.

Budden, K. G.: The theory of the radio window in the ionosphere and magnetosphere, J. Atmos. Terr. Phys., 42, 287-198, 1980.

Carpenter D. L. and Anderson R. R.: An ISEE/Whistler model of equatorial electron density in the magnetosphere, J. Geophys. Res., 97, 1097-1108, 1992.

Christiansen, P. J., Etcheto, J., Ronmark, K., and Stenflo, L.: Upper hybrid turbulence as a source of nonthermal continuum radiation, Geophys. Res. Lett, 11, 139-142, 1984.

Calvert, W.: DE-1 measurements of AKR wave directions, Geophys. Res. Lett., 12, 381-384, 1985.

Darrouzet, F., Décréau, P. M. E., De Keyser, J., Masson, A., Gallagher, D. L., Santolik, O., Sandel, B. R., Trotignon, J.-G., Rauch, J.-L., La Guirriec, E., Canu, P. Sedgemore, F., André, M., and Lemaire, J. F.: Density structures inside the plasmasphere: Cluster observations, Ann. Geophys., 22, 2577-2585, 2004.

Décréau, P. M. E., Fergeau, P., Krasnoselskikh, V., Le Guirriec, E., Lévêque, M., Martin, P., Randriamboarison, O., Rauch, J. L., Sené, F. X., Séran, H. C., Trotignon, J. G., Canu, P., Cornilleau, N., de Féraudy, H., Alleyne, H., Yearby, K., Mögensen, P. B., Gustafsson, G., André, M., Gurnett, D. C., Darrouzet, F., Lemaire, J., Harvey, C. C., Travnicek, P., and Whisper experimenters: Early results from Whisper instrument on Cluster: an overview, Ann. Geophys., 19, 1241-1258, 2001, http://www.ann-geophys.net/19/1241/2001/.

Décréau, P. M. E., C. Ducoin, C., Le Rouzic, G., Randriamboarison, O., Rauch, J. L., Trotignon, J., G., Vallieres, X., Canu, P., Darrouzet, F., Gough, M. P., Buckley, A. M., and Carrozi, T. D.: 
Observation of Continuum radiations from the CLUSTER fleet: first results from direction finding, Ann. Geophys., 22, 26072624, 2004, http://www.ann-geophys.net/22/2607/2004/.

Décréau, P. M. E., Le Guirriec, E., Rauch, J. L., Trotignon, J., G., Canu, P., Darrouzet, F., Lemaire, J., Masson, A., Sedgemore, F., André, M.: Density irregularities in the plasmasphere boundary layer: Cluster observations in the dusk sector, Adv. Space Res., 36, 1964-1969, doi:10.1016/j.asr.2005.08.050, 2005.

Etcheto, J., Christiansen, P. J., Gough, M. P., and Trotignon, J. G.: Terrestrial continuum radiation observations with GEOS-1 and ISEE-1, Geophys. Res. Lett., 9, 1239-1242, 1982.

Gough, M. P., Christiansen, P. J., Martelli, G., and Gershuny, E. J.: Interaction of electrostatic waves with warm electrons at the geomagnetic equator, Nature, 279, 515-517, 1979.

Gough, M. P.: Non-thermal continuum emissions associated with electron injections: remote plasmapause sounding, Planet. Space Sci., 30, 657-668, 1982.

Gurnett, D. A.: The earth as a radio source: the nonthermal continuum, J. Geophys. Res., 80, 2751-2763, 1975.

Jones, D.: Source of terrestrial non-thermal radiation, Nature, 260, 686-689, 1976.

Jones, D.: Latitudinal beaming of planetary radio emissions, Nature, 288, 225-229, 1980.

Jones, D.: First remote sensing of the plasmapause by terrestrial myriametric radiation, Nature, 294, 728-730, 1981.

Jones, D.: Terrestrial myriametric radiation from the earth's plasmapause, Planet. Space Sci., 30, 399-410, 1982.
Jones, D.: A technique for studying density gradients and motions of plasmaspheric irregularities, J. Geophys., 52, 158-166, 1983.

Jones, D., Calvert, W., Gurnett, D. A., and Huff, R. L.: Observed beaming of terrestrial myriameric radiation, Nature, 328, 391395, 1987.

Kasaba, Y., Matsumoto, H., Hashimoto, K., Anderson, R. R., Bougeret, J. L., Kaiser, M. L., Wu, X. Y., and Nagano, I.: Remote sensing of the plasmapause during substorms: Geotail observation of nonthermal continuum enhancement, J. Geophys. Res., 103, 20389-20 405, 1998.

Kennel, C. F., Scarff, F. L., Fredericks, R. W., McGehee, J. H., and Coroniti, F. V.: VLF electric field observations in the magnetosphere, J. Geophys. Res., 75, 6136-6152, 1970.

Kurth, W. S., Gurnett, D. A., and Anderson, R. R.: Escaping nonthermal continuum radiation, J. Geophys. Res., 96, 5519-5531, 1981.

Kurth, W. S.: Continuum radiation in planetary magnetospheres, Planetary Radio Emission III, edited by: Rucker, H. O., Bauer, S. J., and Kaiser, M. L., pp 329-350, Austrian Acad. Of Sci. Press, Vienna, 1992.

Morgan, D. D. and Gurnett, D. A.: The source location and beaming of terrestrial Continuum radiation, J. Geophys. Res., 96, 95959613, 1991.

Rönnmark, K.: Generation of magnetospheric radiation by decay of Bernstein waves, Geophys. Res. Lett., 12, 639-642, 1985.

Tsyganenko, N. A.: Global quantitative models of the geomagnetic field in the cislunar magnetosphere for different disturbance levels, Planet. Space Sci., 35, 1347-1358, 1987. 